\title{
Desarrollo de una tecnología del procesamiento de frijol en polvo deshidratado
}

\author{
Walter Lenin Espinoza Vanegas ${ }^{1}$
}

\section{RESUMEN}

Esta investigación desarrolla una tecnología para el procesamiento de frijol en polvo deshidratado. Esta investigación tiene un enfoque mixto, cuali-cuantitativa. Se aplicó una encuesta que abarca aptitudes de aceptación, preferencia en presentaciones del producto, frecuencia de compra, el consumidor, aspectos demográficos y competencia. Se realizó un análisis univariado de las cinco repeticiones del flujo de proceso obtenido en fase 3, para evaluar el balance de masa del prototipo, en los distintos pasos del flujo de proceso final: remojo, cocción, molido, deshidratado, molienda seca, prototipo final. Los principales resultados muestran que el rendimiento de frijol crudo hasta el producto final deshidratado es de $40.72 \%$ y al rehidratar aumenta al $181 \%$, demostrando que no hay perdida del producto al aplicar la tecnología de deshidratación. La investigación expone la aceptación del producto en el mercado, el 90\% de las personas encuestadas estar dispuesta a consumir frijoles deshidratados. Del $90 \%$ de interesados, un $60 \%$ comprarían el producto ocasionalmente y el 40\% frecuentemente. El $80 \%$ de los encuestados gustaron del sabor de los frijoles, el 15\% mejorarían su consistencia, el 3\% le aumentaría la concentración de sal, y el 2\% mejorarían su textura.

Palabras claves: Tecnología, frijol, procesamiento

Recibido: 3 de marzo de 2016

Aceptado: 4 de abril de 2016

1 UNAN-Managua/FAREM-Estelí. Correo Electrónico: walespinoza2003@yahoo.com 


\title{
Development of a technology of processing dehydrated bean powder
}

\begin{abstract}
This research develops a technology for processing dehydrated bean powder. This research has a mixed approach, quantitative and qualitative. A survey covering skills of acceptance, product presentations preference, purchase frequency, consumer demographics and competition was applied. A univariate analysis of five repetitions of the process flow obtained in Phase 3 was conducted to evaluate the mass balance of the prototype, in the different steps of the flow of final process: soaking, cooking, grinding, dehydrated, dry grinding, and final prototype. The main results show that the yield of raw beans to the final dehydrated product is rehydrated $40.72 \%$ and increases to $181 \%$, demonstrating that there is no loss of the product by applying dehydration technology. The research exposes product acceptance in the market, 90\% of respondents being willing to consume dried beans. $90 \%$ of stakeholders, $60 \%$ occasionally buy the product and $40 \%$ frequently. $80 \%$ of respondents liked the taste of the beans, $15 \%$ would improve their consistency, $3 \%$ will increase the salt concentration, and $2 \%$ improve texture.
\end{abstract}

Keywords: Technology, bean, processing. 


\section{INTRODUCCIÓN}

Las leguminosas de grano se han reconocido como fuente importante de proteína en la dieta de las poblaciones de muchas regiones del mundo. De acuerdo a la cantidad de consumo, las leguminosas pueden cubrir la necesidad humana de ingesta de proteína, pero además pueden proveer la calidad proteica deseada para la alimentación de grupos de poblaciones vulnerables, como los infantes, niños, madres embarazadas y lactantes, cuando se consumen en cantidades adecuadas, Garcia (2003). El grano de frijol es un componente esencial en la dieta de la mayoría de la población rural, y en algunos casos de la urbana, de los países productores más pobres y en vías de desarrollo (Rosas, 2002). Es una de las fuentes principales de proteínas para la mayoría de la población Nicaragüense, donde se prefiere el frijol rojo de variedades que se cultivan en el país.

Garcia (2003) hace referencia a Viana (1999) donde menciona que el uso de variedades de frijol en Nicaragua ha cambiado radicalmente, de modo que las de uso común y más recomendables a sembrar son: Los INTA (Norte, Rojo, Vaina Roja, Sequia, Zamorano, Tomabú). Los tipos de procesamiento a los cuales se somete este producto deben desarrollarse de acuerdo con los hábitos dietéticos de la población y con la forma de consumo del alimento. Asimismo, el tecnólogo al preparar el producto deberá tener en mente la conveniencia de las amas de casa. Por otra parte, vale destacar que el cambio en el estilo de vida a raíz del ingreso de la mujer a la fuerza laboral y el desarrollo de las ciudades, ha impulsado a la creación de nuevos productos alimenticios. En la sociedad aumenta la necesidad de consumir productos de fácil preparación y conservación, que tengan una mayor vida útil en las estanterías de los supermercados.

Los productos de conveniencia son productos de consumo que suelen adquirirse de inmediato y requieren un mínimo de esfuerzo de compra y preparación. Estos productos son colocados por los productores en lugares donde los clientes los adquieren fácilmente.

La industria alimentaria tiene la difícil labor de desarrollar proyectos de investigación y desarrollo de nuevos productos que beneficien al consumidor, tanto en ahorro de tiempo como en la percepción sentida al ingerir alimentos que son semejantes o iguales a los elaboradas en la cocina del hogar. Los productos deshidratados para uso instantáneo que puedan almacenarse por largos períodos de tiempo y mantener los atributos sensoriales propios del alimento, es uno de esos retos.

El frijol es uno de los alimentos básicos dentro de la dieta del nicaragüense, pero su preparación laboriosa requiere energía y un tiempo de cocción muy elevado, las mujeres que tradicionalmente se han dedicado a las labores hogareñas están optando cada vez más por laborar fuera de casa, lo que exige la disponibilidad de alimentos de fácil preparación final y que brinden la ingesta necesaria de proteínas, actualmente existe en mercado alternativas al consumidor pero su alto costo no permite que este producto sea del consumo regular en los hogares de clase media y baja.

Durante el presente año el INTA Región I desarrollara a nivel de campo y laboratorio un producto para uso instantáneo a base de frijol, el cual será cocido, conservado y empacado. En los últimos años ha existido una creciente oferta de frijoles cocidos, licuados y, congelados en las cadenas de supermercados en Estelí y Ocotal (Mendoza, 2003). Dadas estas condiciones de mercado, el prototipo el INTA parece ser muy prometedor; sin embargo, existe la necesidad de refinarlo y de diseñar un sistema eficiente de producción.

En Nicaragua se cultivan diferentes variedades de frijol, pero a estos se les da poco proceso de agro trasformación debido al escaso desarrollo de tecnología 
en este rubro. Cabe destacar que en el país el valor agregado que se le da al frijol consiste en operaciones de limpieza y empacado de estas leguminosa, se le atribuye este problema al el bajo nivel tecnológico de trasformación.

En artículos publicados por el diario nicaragüense "La Prensa "en la fecha 07/06/2014 se menciona que en Nicaragua se necesita que los productores, en forma de cooperativas o individuales, den valor agregado al frijol. De acuerdo con este artículo se tiene que alargar su cadena agroindustrial, agregándole valor. Se tiene que mencionar que para conservar este grano se utilizadas aditivos tales como fosfuro de aluminio el que es un compuesto altamente tóxico. Además, el frijol en su proceso de preparación requiere de mucha energía térmica para su cocción.

En los últimos años ha existido una creciente oferta de frijoles cocidos, licuados y congelados en las cadenas de supermercados de Estelí de marcas nacionales como gallito y extranjeras como es Natura, pero su costo no permite que ese producto sea de consumo regular. Existe la necesidad de procesar el frijol en una forma que sea fácil su almacenamiento y no requiera aditivos para su conservación pero es necesario diseñar un sistema eficiente de agro transformación.

La técnica de conservación preserva la calidad de los alimentos bajando la actividad de agua (aw) mediante la disminución del contenido de humedad, evitando así el deterioro y contaminación microbiológica de los mismos durante el almacenamiento. Para ello se pueden utilizar varios métodos de deshidratación o combinación de los mismos, tales como secado solar, aire caliente, microondas, liofilización, atomización, deshidratación osmótica, entre otros.

Para obtener alimentos deshidratados de buena calidad es imprescindible estudiar en detalle los fenómenos de transferencia de materia y energía involucrados en el proceso, como los cambios producidos a nivel estructural (porosidad, firmeza, encogimiento, densidad) y las reacciones bioquímicas que se llevan a cabo en el momento del proceso (oxidación, enzimáticas, no enzimáticas, desnaturalización).

Desde el punto de vista comercial una importante ventaja de utilizar esta técnica, es que al convertir un alimento fresco en uno procesado (deshidratado) se añade valor agregado a la materia prima utilizada. Además se reducen los costos de transporte, distribución y almacenaje debido a la reducción de peso y volumen del producto en fresco.

Sin embargo, algunos alimentos deshidratados enteros, en trozos o pulverizados, deben ser rehidratados para su consumo o uso posterior en diferentes procesos. Es por ello que el estudio de la transferencia de materia ocurrida durante el fenómeno de rehidratación es importante. Cabe señalar que la rehidratación no es el proceso inverso a la deshidratación, ya que ambos fenómenos tienen diferentes mecanismos de transferencia de materia y dependen de factores distintos. Las operaciones previas a la deshidratación, llamadas pretratamientos, tienen marcada influencia sobre las características y la composición del producto finalmente rehidratado. Aquellos pretratamientos que contribuyen a mantener la integridad de los tejidos permiten evitar mayores pérdidas de sólidos solubles hacia el medio de rehidratación.

El objetivo de esta investigación es desarrollar una tecnología de procesamiento de frijol cocido deshidratado y empacado para uso instantáneo y mejorar la eficiencia de su producción. Para ello se desarrolló una formulación y un procedimiento que lleven a un producto aceptado por el cliente potencial, y un balance de materia del proceso de elaboración de frijol cocido deshidratado.

\section{El proceso de industrialización del frijol}

La industrialización del frijol podría constituir una manera indirecta de incrementar su cultivo, a través 
de un incentivo económico más estable. Además, el producto procesado tendría las ventajas de dar mayor estabilidad, disponibilidad constante a través de todo el año, más uniformidad, más fácil preparación, y podría ser un vehículo para otros nutrientes.

El secado de granos se puede definir de distintas maneras, según el enfoque que se desee adoptar. En los estudios más teóricos se pone el énfasis en los mecanismos de transferencia de energía y de materia. Así, el secado se puede definir como un proceso en que hay intercambio simultáneo de calor y masa, entre el aire del ambiente de secado y los granos. En cambio, en los casos generales, se define el secado como la operación unitaria responsable de la reducción del contenido de humedad de cierto producto, hasta un nivel que se considera seguro para el almacenamiento de éste.

Se entiende que es seguro un nivel de humedad por debajo del cual se reduce la actividad respiratoria de los granos y se dificulta el ataque de insectos y hongos. Dicho nivel varia con los distintos tipos de granos, pero, para las condiciones brasileñas y los granos más comunes, abarca una gama entre 10 y $14 \%$ de humedad expresada sobre base húmeda.

Al llegar a la madurez fisiológica, los productos agrícolas y en especial los granos, presentan en la mayoría de los casos, un contenido máximo de materia seca. Sería recomendable cosechar los granos en ese punto de desarrollo, desde el punto de vista del rendimiento máximo de la producción. No obstante, varios factores limitan esa posibilidad, destacándose entre ellos el contenido de humedad de los granos.

Al comienzo de la etapa de madurez fisiológica, los granos presentan, además del contenido máximo de materia seca, un elevado porcentaje de agua. Esta condición acarrea algunas dificultades, pues los granos no resisten los procedimientos mecánicos de recolección y demás operaciones de tratamiento. Es preciso esperar, entonces, que la cosecha se realice "tan pronto" como los granos lo soporten. Esta técnica ofrece algunas ventajas, como son: un mayor porcentaje de materia seca (como ya se mencionó), menor contaminación del producto en el campo, menor pérdida por ataque de depredadores, mayor porcentaje de germinación y vigor y otras.

El mayor inconveniente de la cosecha de productos húmedos es la necesidad de reducir su contenido de humedad a niveles seguros para su almacenamiento, siendo un proceso que exige un gran consumo de energía. En general el secado artificial de granos es una técnica poco difundida en América Latina. En las regiones en que se la conoce, normalmente está mal utilizada. En consecuencia, hay elevadas pérdidas de granos en esos países.

El continuo adelanto en la tecnología de alimentos ha permitido desarrollar diferentes métodos de preparación de frijoles enteros pre cocidos y deshidratados. La mayor parte de la investigación ha sido enfocada a la disminución del tiempo de cocción y a la vez evitar cambios adversos en las características físicas y sensoriales del producto final, como lo son la textura, color, olor y sabor.

Según (Barbosa G, 2000) en líneas generales, uno de éstos métodos consiste en someter los frijoles a remojo, cocerlos a presión y deshidratarlos (Feldberg et al, 1956). La etapa de escaldado ha sido propuesta por algunos investigadores (Dorsey et al, 1961), porque este tratamiento adicional ofrece las ventajas de garantizar la hidratación completa e inactivación de la lipooxidasa, afianzando así la estabilidad del producto procesado durante el almacenamiento.

El congelamiento antes o después de la cocción, así como el recubrimiento con una capa de azúcares, tiene como finalidad evitar las fisuras que se presentan en el grano de frijol cocido. Los tiempos de cocción del producto final varían de acuerdo con la variedad de 
frijol usada (Cuadro 4), con el método de preparación, así como las condiciones previas de conservación. En este caso el producto final está listo para el consumo después de rehidratarlo y cocerlo por 5 a 10 minutos.

El principal objetivo es obtener un producto de cocimiento rápido con el mínimo de deterioro de las características sensoriales y nutricionales de la materia prima. Con respecto a las propiedades físicas, dos han sido los problemas encontrados: la textura del producto final y la decoloración de la harina. La pérdida parcial del color se da cuando se emplea frijol negro. Este es un aspecto muy importante desde el punto de vista del consumidor que asocia la buena calidad de la sopa con el color más oscuro. La textura más espesa se debe a la presencia de partículas secas de la cáscara que no están totalmente pulverizadas (Rojas, 2002)

\section{Propiedades sensoriales}

El continuo adelanto en la tecnología de alimentos ha permitido desarrollar diferentes métodos de preparación de frijoles enteros pre cocidos y deshidratados. La mayor parte de la investigación ha sido enfocada a la disminución del tiempo de cocción y a la vez evitar cambios adversos en las características físicas y sensoriales del producto final, como lo son la textura, color, olor y sabor.

En líneas generales, uno de éstos métodos consiste en someter los frijoles a remojo, cocerlos a presión y deshidratarlos (Feldberg et al, 1956). La etapa de escaldado ha sido propuesta por algunos investigadores (Dorsey et al, 1961), porque este tratamiento adicional ofrece las ventajas de garantizar la hidratación completa e inactivación de la lipooxidasa, afianzando así la estabilidad del producto procesado durante el almacenamiento.

Todos los alimentos tienen características particulares que determinan su calidad. La calidad se relaciona con el grado de cumplimiento de las expectativas del consumidor y está relacionada con características físicas, químicas y biológicas. Los consumidores son los que tienen la última palabra al momento de la compra. Siempre deben controlar que el alimento no luzca sospechoso, chequear que esté intacto, que no esté vencido, hinchado, que esté en buen estado y que su aspecto sea el de un producto fresco, limpio y de buena calidad.

Las propiedades sensoriales de un alimento son las que se pueden percibir con los sentidos; la vista, el olfato y el gusto son los sentidos más usados, ya que con ellos se determina si un alimento luce fresco o sospechoso. Con el tacto se percibe la textura, firmeza, humedad, superficie lisa-pegajosa-brillante. El gusto ayuda a detectar si el sabor es rancio, ácido, salado, dulce, etc. Este es el último sentido que se utiliza, ya que a veces puede evitar un riesgo para la salud. La dificultad radica en que los gustos son muy personales, aunque los factores culturales pueden marcar tendencias.

Hay dos tipos de análisis sensorial que se pueden realizar sobre productos alimenticios: los que se centran en las características de los alimentos (pruebas sensoriales analíticas) y los que miden las respuestas que ese producto provoca en el consumidor (pruebas sensoriales de preferencia - aceptación)

\section{MATERIALES Y MÉTODOS}

La etapa experimental de esta investigación se realizó en la Universidad Nacional Autónoma de Nicaragua UNAN Managua, en la Facultad Regional Multidisciplinaria (UNAN-Managua; FAREM-Estelí), ubicada en el municipio de Estelí.

Según el tiempo de ocurrencia de los hechos y registros de la información la investigación es un estudio prospectivo, porque se registró la información, según iban ocurriendo los fenómenos. De acuerdo al análisis y alcance de los resultados se clasifica como una investigación descriptiva porque se precisaron las 
situaciones predominantes a través de la descripción exacta de las actividades y procesos.

Las etapas de la investigación abarcaron:

Revisión bibliográfica: Para la obtención de la información se realizaron consultas bibliográficas, se elaboraron el protocolo de investigación que consistirá en la delimitación del tema, objetivos, planteamiento, desarrollo el marco teórico y elaboración del diseño metodológico todo esto se utilizara como base para la elaboración del documento.

Fase Experimental: Para realizar el experimento se utilizaron 25 libras en cada ensayo y se realizaron tres ensayos para un total de 75 libras a utilizar para desarrollar la tecnología de procesamiento de frijol en polvo deshidratado.

En la primera fase experimental, se realizaron tres ensayos del flujo de proceso inicial, para validar los resultados obtenidos y determinar posibles cambios en el proceso tentativo, que hagan el proceso más eficiente. La segunda fase experimental abarcó los procesos de remojo, cocción, molido, deshidratado, molienda seca y empaque. Para cada etapa se determinó el tiempo, cantidad de materia prima ingresando al proceso en cada etapa, temperaturas y tiempos de procesos específicos como cocción y deshidratado.

- Para determinar el sabor del producto: Fue utilizada sal y ajo al 1\%, al obtener el prototipo final se evaluara mediante análisis sensoriales y degustaciones, si el sabor es agradable o no al consumidor.

- Para definir el tiempo de cocción: Se evaluaron en la autoclave tres tiempos de cocción, 30, 35 y 40 minutos. Las muestras fueron degustadas por grupos focales constituidos por ocho señoras que frecuentemente preparan frijol.

- Para definir el tiempo de deshidratación: Se evaluaron dos tiempos de deshidratación, 6 y 8 horas.
Los instrumentos utilizados fueron: balanzas de reloj para pesar la cantidad de libras de granos de frijol que entran y las que salen del deshidratador, reloj digital para determinar el tiempo de deshidratación de los granos, hoja de toma de datos para registrar los datos, termómetros de espiga selladora térmica. Los materiales: frijoles, agua sal, ajo, bolsas laminadas. Los equipos: autoclave, colador, cucharones deshidratador, y licuadora.

Se utilizaron los siguientes ingredientes: frijol agua, ajo, y sal. El equipo usado está: marmita, recipientes de acero inoxidable, procesador de alimentos, bolsas plásticas, deshidratador, y molino. El procedimiento que se aplicó fue el siguiente:

1. Seleccionar y lavar el frijol.

2. Pesar los ingredientes.

3. Poner a cocer con agua suficiente para cubrir los frijoles, añadir ajo y sal hasta darle el sabor deseado; el fríjol debe retirarse cuando al presionarlo suavemente con los dedos se desbarate por completo. Al utilizar la marmita para cocer los frijoles, el agua evaporada debe restaurarse continuamente para evitar que los frijoles se quemen.

4. Licuar los frijoles hasta formar una pasta.

5. Colocar la pasta de frijoles en la bandeja del deshidratador, con velocidad $2 \mathrm{~m} / \mathrm{s}$ durante seis horas a $73.15^{\circ} \mathrm{C}$.

6. Cuando el fríjol esté seco molerlo en el molino hasta obtener polvo de frijol.

\section{El análisis y procesamiento de la información}

Se realizó un análisis univariado de las cinco repeticiones del flujo de proceso obtenido en fase 3, para evaluar el balance de masa del prototipo, en los distintos pasos del flujo de proceso final: remojo, cocción, molido, deshidratado, molienda seca, prototipo final. Para elaborar el documento se utilizó el programa Word 2010, y para el análisis y procesamiento de datos se 
aplicaron los programas: Excel 2010, InfoStatf y SPSS. Se aplicó una encuesta a consumidores del puesto de Ventas y supermercado Maxi Pali de Estelí y Pali de Ocotal para determinar el análisis sensorial. La encuesta fue estructurada con el objetivo de recolectar información acerca del mercado de productos de frijol de rápida preparación. La encuesta abarca aptitudes de aceptación, preferencia en presentaciones del producto, frecuencia de compra, el consumidor, aspectos demográficos y competencia. El número de encuestas y de gustaciones a aplicarse fue definido por el análisis estadístico hecho a una prueba piloto. La misma encuesta fue aplicada después a la muestra final.

La encuesta se diseñó con 13 preguntas, las primeras ocho se refieren a información general y las otras cinco al análisis de degustación. Todas las muestras fueron presentadas de forma simultánea. Se mostraron dos muestras de frijol, una fue el prototipo obtenido y la otra fue un producto de marca Natura. Se determinó con la población la aceptabilidad en cuanto a textura, sabor y apariencia general.

Los resultados fueron evaluados utilizando la tabla para 3-100 panelistas y 3-12 muestras. Las diferencias entre todos los posibles pares se comparan con el valor crítico de la tabla, en base a un nivel de significancia determinado (95\%), y al número de panelistas y muestras empleadas en la prueba.

\section{RESULTADOS Y DISCUSIÓN}

A partir del estudio se determinó el proceso de elaboración de frijoles cocidos, molidos y deshidratados para uso instantáneo, y se describe a continuación:

- Tipo: Disponer de frijol rojo variedad una humedad de $11.5 \%$, y llevarlos a un recipiente su posteriormente colocar en remojo.

- Selección: Eliminar granos dañados y otras partículas que estén contaminando el producto.
- Lavado: Colocar el producto en un colador y lavar hasta eliminar todas las impurezas del ambiente o residuos que pudieron quedar después del proceso de selección.

- Remojo: Colocar los frijoles con agua y dejar reposar durante seis horas.

- Preparación del equipo: Lavar el autoclave, las licuadoras y las paletas que van a ser utilizados a partir de este momento.

- Cocción: Colocar en un recipiente el frijol, agua y ajo. Someter en autoclave los ingredientes a cocción durante 40 minutos a $115^{\circ} \mathrm{C}$.

- Molido: Retirar cuidadosamente el recipiente con los frijoles cocidos, retirar el agua de cocción y pesar frijoles y agua por separado. Llevar a la licitadora y preparar el equipo con un colador, colocar los frijoles y la mitad del agua de cocción. Posterior se obtuvieron dos masas de diferente concentración; para uniformizar el puré.

- Deshidratado: Sobre las bandejas distribuir el producto uniformemente, esparciéndolo con ayuda de una paleta y formando una masa de no más $1.5 \mathrm{~cm}$ de espesor. Cerrar correctamente el deshidratador.

- Molido: Retirar las bandejas después de terminado el tiempo de deshidratación y colocar el producto en bolsas plásticas para evitar el intercambio de humedad con el ambiente. Llevar el producto a molienda seca. Licuar el producto por tandas, llenando el envase hasta tres cuartas partes de su contenido total. Se obtiene un producto fino, que se pasa por un colador de malla de $0.33 \mathrm{~cm}$, para eliminar partículas de mayor tamaño.

- Empacado: El producto se coloca en bolsas laminadas correctamente selladas, para preservar el producto de la humedad del ambiente.

- Rehidratado: Se recomienda añadir agua en su punto de ebullición, con una proporción de 1 litro de agua por cada $454 \mathrm{~g}$ de producto. El consumidor final puede añadir más o menos agua dependiendo de su preferencia. El aumento en peso es de 3.2 veces el peso original. 
Los tiempos de elaboración del producto en cada etapa del proceso se observan en el Cuadro No.1 y son:

Cuadro 1. Tiempos en cada paso del proceso para la elaboración del prototipo de frijol cocido, molido, deshidratado, en tandas de $2 \mathrm{~kg}$.

\begin{tabular}{lr}
\hline \multicolumn{1}{c}{ Procedimiento } & Tiempo (h) \\
\hline Remojo & 6 \\
Cocción & 1.5 \\
Molienda & 1 \\
Deshidratado & 8 \\
Molienda seca & 3.5 \\
Empacado & 1 \\
\hline
\end{tabular}

Fuente: Resultados de la etapa experimental del estudio.

Se aplicó una encuesta a consumidores potenciales para valorar el consumo actual de productos existentes en el mercado y la posible aceptación del prototipo. Los resultados fueron evaluados considerando la información general sobre el tipo de productos que consumen y existen en el mercado, su compra y preferencia, y por otra parte la degustación para que el consumidor estuviera expuesto al producto y tuviera suficientes criterios para decidir o no su compra.

Se encuestaron a 205 personas, el $17 \%$ son estudiantes, $56 \%$ profesionales y el $28 \%$ ejercen un oficio. El $28 \%$ de los consumidores prefieren consumir frijoles cocidos y el $72 \%$ molidos. Los encargados de comprar los frijoles son: $84 \%$ las amas de casa, $22 \%$ los hombres y un $2 \%$ otros miembros de la familia y empleados.

En relación al proceso de elaboración de los frijoles en sus casas, los condimentos preferidos al cocer los frijoles son: ajo y sal. Para freír se prefiere utilizar: cebolla, chile y apio. El 37\% de los encuestados compran frijoles listos para consumir, $50 \%$ los preparan en sus hogares el restante $13 \%$ no frecuentan este tipo de productos. Las marcas de mayor posicionamiento en el mercado son: Naturas $94 \%$, el gallito $6 \%$. La prueba de degustación dio los siguientes resultados:
El $90 \%$ estaría dispuesto a consumir frijoles deshidratados, el 10\% restante no está interesado. Del $90 \%$ de los interesados; el $60 \%$ comprarían el producto ocasionalmente y el $40 \%$ frecuentemente. Al $80 \%$ le gustaron del sabor de los frijoles, el $15 \%$ mejorarían su consistencia, el 3\% le aumentaría la concentración de sal, el 2\% mejorarían su textura.

El $75 \%$ prefiere un empaque de aluminio, el $22 \%$ prefieren un empaque plástico y el $3.12 \%$ recipientes rígidos. La cantidad adecuada para este producto fue de 454 gramos para el 69\%, 227 gramos el 6.25\% y más de 454 gramos el $6 \%$.

\section{Análisis sensorial}

El prototipo molido en licuadora tuvo total aceptación en los tres factores evaluados, lo cual indica que es necesario moler el frijol hasta obtener una harina fina, para mejorar la textura del producto final rehidratado. La cantidad de agua para rehidratación fue igual para las dos muestras. Sin embargo, los participantes del panel sensorial opinaron sobre hacer la pasta más liviana, este factor puede modificarse añadiendo mayor cantidad de agua al producto. La consistencia final va a depender de los gustos y preferencias del consumidor.

\section{Balance de materia}

Después de recopilar los datos obtenidos en las cinco repeticiones, se realizó un análisis univariado (Anexo 4). Los resultados fueron los siguientes:

El balance de masa en el proceso de remojo: Se pesaron $2.09 \mathrm{~kg}$ de frijol crudo y $8.06 \mathrm{~kg}$ de agua para remojar los frijoles. Después del tiempo de remojo los granos de frijol se hinchan, y su peso fue de $3.06 \mathrm{~kg}$. El agua que se usó para el remojo se pesó y el valor obtenido fue $6.96 \mathrm{~kg}$.

El balance de masa en el proceso de cocción: Se utilizaron $3.05 \mathrm{~kg}$ de frijol y $6.86 \mathrm{~kg}$ de agua para cocer 
los frijoles. Las cantidades de ajo y sal a añadirse fueron de 0.098 y $0.096 \mathrm{~kg}$, respectivamente. Después de cocción los frijoles tuvieron un peso promedio de $4.46 \mathrm{~kg}$ y el agua de cocción de $4.96 \mathrm{~kg}$. La mitad del agua de cocción $(2.48 \mathrm{~kg})$, se utilizó para elaborar el puré durante el proceso de molienda.

\section{El balance de masa en el proceso de molienda:} En la licuadora entraron $4.46 \mathrm{~kg}$ de frijol cocido y $2.48 \mathrm{~kg}$ de agua. Durante el proceso de molienda una cantidad de puré de frijol queda adherida en el equipo y no disponible para seguir en el proceso. Al trabajar con tandas más grandes, las mermas son menores, incrementando el rendimiento por $\mathrm{kg}$ de frijol crudo. Después de pasar por le despulpador se obtuvieron en promedio $5.82 \mathrm{~kg}$ de puré para ser deshidratado.

El balance de masa en el proceso de deshidratación: Después de deshidratación se obtuvieron $1.22 \mathrm{~kg}$ de frijol deshidratado.

\section{El balance de masa en el proceso de molienda:} Después de licuar $1.22 \mathrm{~kg}$ de frijol, se obtuvieron en promedio $1.18 \mathrm{~kg}$ de prototipo final. Los $0.04 \mathrm{~kg}$ que se pierden durante esta parte del proceso, se deben principalmente a la volatilidad del producto después de licuado. Los rendimientos de frijol fueron de 59\%. Este resultado pueden deberse a varios factores de transformación de la materia prima durante el proceso, como son: la cocción, molido y el deshidratado.

\section{Rendimiento del prototipo:}

Rendimiento $=1.18 \mathrm{~kg}$ del prototipo final $/ 2.05 \mathrm{~kg} \mathrm{de}$ frijol crudo $=59 \%$

Si se deshidrata el producto final el rendimiento es de $188 \%$ con respecto a la materia prima inicial y la cantidad final del producto ya rehidratado.

Rendimiento $=5.76 \mathrm{~kg}$ del prototipo final rehidratado / $2.05 \mathrm{~kg}$ de frijol crudo $=188 \%$

\section{Remojo:}

Frijol crudo $=27.6 \mathrm{~kg}$ Frijol remojado $=40.47 \mathrm{~kg}$ Rendimiento $=146 \%$

Se tiene un rendimiento de $146 \%$, de frijol crudo a frijol después de remojo.

\section{Cocción:}

Frijol crudo $=27.6 \mathrm{~kg}$

Frijol cocido $=59.18 \mathrm{~kg}$

Rendimiento $=214 \%$

El rendimiento de frijol después de cocido es de $214 \%$.

\section{Molido:}

Frijol crudo $=27.6 \mathrm{~kg}$ Puré $=77.22 \mathrm{~kg}$ Rendimiento $=$ $279 \%$

El rendimiento del frijol crudo hasta llegar al puré obtenido durante la molienda en la despulpadora es de $279 \%$.

\section{Molido en seco:}

Frijol crudo $=27.6 \mathrm{~kg}$

Frijol deshidratado $=11.24 \mathrm{~kg}$

Rendimiento $=40.72 \%$

El rendimiento de frijol crudo hasta el producto final deshidratado es de $40.72 \%$.

\section{Rehidratación:}

Frijol crudo $=27.6 \mathrm{~kg}$

Prototipo rehidratado $=50.10$

Rendimiento $=181.52 \%$

Al momento de rehidratar el producto el rendimiento es de $181.52 \%$, considerando $27.6 \mathrm{~kg}$ de frijol crudo entrando al proceso.

Finalmente, la formulación del producto (prototipo) se define con las siguientes características:

Cuadro No.2. Formulación del Producto.

\begin{tabular}{lr}
\hline \multicolumn{1}{c}{ Ingredientes } & Porcentajes (\%) \\
\hline Frijoles remojados & 30.29 \\
Agua & 67.88 \\
Ajo & 0.97 \\
Sal & 0.95
\end{tabular}




\section{CONCLUSIONES}

- El prototipo de frijol cocido, molido, deshidratado fue de amplia aceptación en la población encuestada, el 90\% estaría dispuesto a adquirir el producto. Este producto tuvo mayor aceptación que el producido por la marca Natura

- Se diseñó el flujo de proceso para la elaboración de frijol cocido, molido, deshidratado.

- Se realizó un balance de materia de las distintas etapas del proceso de agro transformación del frijol demostrando que el rendimiento el rendimiento de frijol crudo hasta el producto final deshidratado es de $40.72 \%$. y al momento de rehidratar es de $181 \%$, es decir que no hay perdida del producto al momento de aplicar la tecnología de deshidratación

- El proceso de elaboración del prototipo de frijol cocido, molido deshidratado requiere remojo durante 6 horas, cocción durante 40 minutos a $115^{\circ} \mathrm{C}$ molienda, deshidratado a $73.15^{\circ} \mathrm{C}$, velocidad de aire de $2 \mathrm{~m} / \mathrm{s}$ durante 8 horas, licuado en seco y empacado.

- La formulación del producto consiste en: agua $67.88 \%$, frijoles $30.2 \%$, sal $0.97 \%$ y ajo $0.95 \%$.

\section{RECOMENDACIONES}

- Probar diferentes temperaturas, velocidades y tiempos de deshidratación que puedan hacer al proceso más eficiente

- Realizar una prueba de mercado y un análisis económico del prototipo.

- Realizar pruebas para prototipos utilizando otros condimentos, como: chile, cebolla, especies.

- Utilizar otros empaques que no sea de aluminio para almacenar el producto.

- Comprobar sensorial y microbiológicamente la vida útil del producto.

\section{RECONOCIMIENTO}

Este estudio se llevó a cabo gracias al financiamiento del Programa de Naciones Unidas (PNUD)

\section{BIBLIOGRAFÍA}

Barbosa G, V. H. (2000). Desidratacion de Alimentos. Zaragoza, España: ACRIBA.

Garcia, C.M.(2003). Una propuesta para la produccion local de semilla de calidad para pequeños agricultores. San Jose ,Costa Rica: PRIAG.

Rojas, L. (2002). FAO Procesamiento. Retrieved Enero 10, 2016, from www.fao.org/inpho/library

Rosas, J. C. (2003). El cultivo del frijol común en América Trópical. Honduras: Zamorano Academic Press.

\section{ANEXOS}

\section{RESULTADOS DEL ANÁLISIS UNIVARIADO}

Cuadro 3: Balance de masa, entradas y salidas durante el proceso de remojoa la entrada de frijol

\begin{tabular}{|l|l|l|l|l|l|}
\hline \multicolumn{2}{|c|}{ Frijoles entrada } & \multicolumn{2}{c|}{ Agua entrada } & \multicolumn{2}{c|}{$\begin{array}{c}\text { Frijoles + Agua } \\
\text { entrada }\end{array}$} \\
\hline Media & 2,09 & Media & 8,06 & Media & 10,13 \\
\hline $\begin{array}{l}\text { Error } \\
\text { típico }\end{array}$ & 0,03 & $\begin{array}{l}\text { Error } \\
\text { típico }\end{array}$ & 0,02 & Error típico & 0,04 \\
\hline Mediana & 2,10 & Mediana & 8,09 & Mediana & 10,10 \\
\hline Moda & 2,10 & Moda & 8,10 & Moda & 10,10 \\
\hline $\begin{array}{l}\text { Desviación } \\
\text { estándar }\end{array}$ & 0,06 & $\begin{array}{l}\text { Desviación } \\
\text { estándar }\end{array}$ & 0,05 & $\begin{array}{l}\text { Desviación } \\
\text { estándar }\end{array}$ & 0,10 \\
\hline $\begin{array}{l}\text { Varianza } \\
\text { de la } \\
\text { muestra }\end{array}$ & 0,00 & $\begin{array}{l}\text { Varianza } \\
\text { de la } \\
\text { muestra }\end{array}$ & 0,00 & $\begin{array}{l}\text { Varianza de } \\
\text { la muestra }\end{array}$ & 0,01 \\
\hline
\end{tabular}

Cuadro 4: Balance de masa, entradas y salidas durante el proceso de remojo a la Salida de frijol

\begin{tabular}{|l|l|l|l|l|l|}
\hline \multicolumn{2}{|c|}{ Frijoles Salida } & \multicolumn{2}{|c|}{ Agua Salida } & \multicolumn{2}{c|}{$\begin{array}{c}\text { Frijoles + Agua } \\
\text { Salida }\end{array}$} \\
\hline Media & 3.06 & Media & 6.86 & Media & 9.91 \\
\hline $\begin{array}{l}\text { Error } \\
\text { típico }\end{array}$ & 0.04 & $\begin{array}{l}\text { Error } \\
\text { típico }\end{array}$ & 0.19 & Error típico & 0.21 \\
\hline Mediana & 3.05 & Mediana & 7 & Mediana & 10,1000 \\
\hline Moda & 3.00 & Moda & 7 & Moda & 10,10 \\
\hline $\begin{array}{l}\text { Desviación } \\
\text { estándar }\end{array}$ & 0.07 & $\begin{array}{l}\text { Desviación } \\
\text { estándar }\end{array}$ & 0.43 & $\begin{array}{l}\text { Desviación } \\
\text { estándar }\end{array}$ & 0,47 \\
\hline $\begin{array}{l}\text { Varianza } \\
\text { de la } \\
\text { muestra }\end{array}$ & 0.01 & $\begin{array}{l}\text { Varianza } \\
\text { de la } \\
\text { muestra }\end{array}$ & 0.19 & $\begin{array}{l}\text { Varianza de } \\
\text { la muestra }\end{array}$ & 0,22 \\
\hline
\end{tabular}


Cuadro 5: Balance de masa, entradas y salidas durante el proceso de cocción de frijol

\begin{tabular}{|l|l|l|l|l|l|}
\hline \multicolumn{2}{|c|}{ Frijoles entrada } & \multicolumn{2}{c|}{ Agua entrada } & \multicolumn{2}{c|}{$\begin{array}{c}\text { Frijoles + Agua } \\
\text { entrada }\end{array}$} \\
\hline Media & 3,05 & Media & 6,86 & Media & 9,91 \\
\hline Error típico & 0,03 & Error típico & 0,19 & Error típico & 0,21 \\
\hline Mediana & 3,00 & Mediana & 7,00 & Mediana & 10,10 \\
\hline Moda & 3,00 & Moda & 7.00 & Moda & 10,10 \\
\hline $\begin{array}{l}\text { Desviación } \\
\text { estándar }\end{array}$ & 0,07 & $\begin{array}{l}\text { Desviación } \\
\text { estándar }\end{array}$ & 0,43 & $\begin{array}{l}\text { Desviación } \\
\text { estándar }\end{array}$ & 0,47 \\
\hline $\begin{array}{l}\text { Varianza de } \\
\text { la muestra }\end{array}$ & $\begin{array}{l}\text { Varianza de } \\
\text { la muestra }\end{array}$ & 0,19 & $\begin{array}{l}\text { Varianza de } \\
\text { la muestra }\end{array}$ & 0,22 \\
\hline
\end{tabular}

Cuadro 6: Balance de masa, de ajo y sal a la entrada de cocción del frijol

\begin{tabular}{|l|l|l|l|l|l|}
\hline \multicolumn{2}{|c|}{ Ajo entrada } & \multicolumn{2}{c|}{ Sal entrada } & \multicolumn{2}{c|}{$\begin{array}{c}\text { Total agua, frijol, } \\
\text { ajo, sal }\end{array}$} \\
\hline Media & 0,098 & Media & 0,096 & Media & 10,10 \\
\hline Error típico & 0,002 & Error típico & 0.002 & Error típico & 0,21 \\
\hline Mediana & 0,100 & Mediana & 0,100 & Mediana & 10,29 \\
\hline Moda & 0,100 & Moda & 0.100 & Moda & \#N/A \\
\hline $\begin{array}{l}\text { Desviación } \\
\text { estándar }\end{array}$ & 0,004 & $\begin{array}{l}\text { Desviación } \\
\text { estándar }\end{array}$ & 0,005 & $\begin{array}{l}\text { Desviación } \\
\text { estándar }\end{array}$ & 0.47 \\
\hline $\begin{array}{l}\text { Varianza } \\
\text { de la } \\
\text { muestra }\end{array}$ & 0.000 & $\begin{array}{l}\text { Varianza de } \\
\text { la muestra }\end{array}$ & 0.000 & $\begin{array}{l}\text { Varianza de } \\
\text { la muestra }\end{array}$ & 0,22 \\
\hline
\end{tabular}

Cuadro 7: Balance de masa de frijoles y agua a la salida de cocción del frijol

\begin{tabular}{|l|l|l|l|l|l|}
\hline \multicolumn{2}{|c|}{ Frijoles } & \multicolumn{2}{c|}{ Agua } & \multicolumn{2}{c|}{ Frijol + Agua } \\
\hline Media & 4,46 & Media & 2,48 & Media & 6,94 \\
\hline Error típico & 0,07 & Error típico & 0,03 & Error típico & 0,07 \\
\hline Mediana & 4,50 & Mediana & 2,50 & Mediana & 7,00 \\
\hline Moda & 4,30 & Moda & 2,50 & Moda & \#N/A \\
\hline $\begin{array}{l}\text { Desviación } \\
\text { estándar }\end{array}$ & 0,15 & $\begin{array}{l}\text { Desviación } \\
\text { estándar }\end{array}$ & 0,06 & $\begin{array}{l}\text { Desviación } \\
\text { estándar }\end{array}$ & 0,16 \\
\hline $\begin{array}{l}\text { Varianza } \\
\text { de la } \\
\text { muestra }\end{array}$ & 0.000 & $\begin{array}{l}\text { Varianza de } \\
\text { la muestra }\end{array}$ & 0.000 & $\begin{array}{l}\text { Varianza de } \\
\text { la muestra }\end{array}$ & 0,22 \\
\hline
\end{tabular}

Cuadro 8: Balance de masa, ala salidas durante el proceso de molido para obtener puré de frijol

\begin{tabular}{|l|l|l|l|l|l|}
\hline \multicolumn{2}{|c|}{$\begin{array}{c}\text { Residuos } \\
\text { (máquina) }\end{array}$} & \multicolumn{2}{c|}{ Puré } & \multicolumn{2}{c|}{ Residuos } \\
\hline Media & 1,12 & Media & 5,82 & Media & 0,40 \\
\hline Error típico & 0,09 & Error típico & 0,09 & Error típico & 0,03 \\
\hline Mediana & 1,10 & Mediana & 5,90 & Mediana & 0,40 \\
\hline Moda & \#N/A & Moda & \#N/A & Moda & 0,40 \\
\hline $\begin{array}{l}\text { Desviación } \\
\text { estándar }\end{array}$ & 0,19 & $\begin{array}{l}\text { Desviación } \\
\text { estándar }\end{array}$ & 0,20 & $\begin{array}{l}\text { Desviación } \\
\text { estándar }\end{array}$ & 0,07 \\
\hline $\begin{array}{l}\text { Varianza de } \\
\text { la muestra }\end{array}$ & 0,04 & $\begin{array}{l}\text { Varianza de } \\
\text { la muestra }\end{array}$ & 0,04 & $\begin{array}{l}\text { Varianza de } \\
\text { la muestra }\end{array}$ & 0,01 \\
\hline
\end{tabular}

Cuadro 9: Balance de masa, entradas y salidas durante el proceso de deshidratación de puré de frijol

\begin{tabular}{|l|l|l|l|}
\hline \multicolumn{2}{|c|}{ Entrada } & \multicolumn{2}{c|}{ Puré } \\
\hline Muré en bandejas & 5,42 & Media & 1,22 \\
\hline Error típico & 0,06 & Error típico & 0,05 \\
\hline Mediana & 5,50 & Mediana & 1,25 \\
\hline Moda & \#N/A & Moda & 1,10 \\
\hline Desviación estándar & 0,14 & Desviación estándar & 0,11 \\
\hline Varianza de la muestra & 0,02 & Varianza de la muestra & 0,01 \\
\hline
\end{tabular}

Cuadro 10: Balance de masa, entradas y salidas durante el proceso de deshidratación de puré de frijol

\begin{tabular}{|l|l|l|l|}
\hline \multicolumn{2}{|c|}{ Entrada } & \multicolumn{2}{c|}{ Puré } \\
\hline \multicolumn{2}{|c|}{ Frijol deshidratado } & Frijol deshidratado molido \\
\hline Media & 1,22 & Media & 1,18 \\
\hline Error típico & 0,05 & Error típico & 0,05 \\
\hline Mediana & 1,25 & Mediana & 1,20 \\
\hline Moda & 1,10 & Moda & $\# \mathrm{~N} / \mathrm{A}$ \\
\hline Desviación estándar & 0,11 & Desviación estándar & 0,10 \\
\hline Varianza de la muestra & 0,01 & Varianza de la muestra & 0,01 \\
\hline
\end{tabular}

